\title{
EVALUATION OF LASER ABLATION MICROTOMY FOR CORRELATIVE MICROSCOPY OF HARD TISSUES.
}

\author{
Alan Boyde \\ Dental Physical Sciences, Queen Mary University of London, London E1 4NS, UK \\ Email: a.boyde@qmul.ac.uk
}

Phone: +44 (0) 2078825984

\begin{abstract}
Laser ablation machining or microtomy (LAM) is a relatively new approach to producing slide mounted sections of translucent materials. We evaluated the method with a variety of problems from the bone, joint and dental tissues fields where we require thin undecalcified and undistorted sections for correlative light microscopy (LM) and backscattered electron scanning electron microscopy (BSE SEM). All samples were embedded in poly-methylmethacrlate (PMMA) and flat block surfaces had been previously studied by BSE-SEM and confocal scanning light microscopy (CSLM). Most were also studied by X-yay microtomography (XMT). The block surface is stuck to a glass slide with cyanoacrylate adhesive. Setting the section thickness and levelling uses inbuilt optical coherence tomographic imaging. Tight focusing of near-infrared laser radiation in the sectioning plane gives extreme intensities causing photodisruption of material at the focal point. The laser beam is moved by a fast scanner to write a cutting line, which is simultaneously moved by an $\mathrm{XY}$ positioning unit to create a sectioning plane. The block is thereby released from the slide, leaving the section stuck to the slide. Light, wet polishing on the finest grade (4000 grit) silicon carbide polishing paper is used to remove a 1-2 $\mu \mathrm{m}$ thick damaged layer at the surface of the section. Sections produced by laser cutting are fine in
\end{abstract}


quality and superior to those produced by mechanical cutting and can be thinner than the 'voxel' in most laboratory X-ray microtomography systems. The present extensive pilot studies have shown that it works to produce samples which we can study by both light and electron microscopy.

Keywords: Osteoporosis, osteoarthritis, enthesis, tendon, ligament, bone

\section{Introduction}

Laser ablation machining or microtomy (LAM) of sections from the front surfaces of plastic embedded tissues has been introduced as a general method for producing sections for light microscopy (LM: Lubatschowski 2007; Richter et al 2011; Will and Richter 2015; Schimek et al. 2013; Kowtharapu et al 2017; Richter et al 2014; Bourassa et al 2016). The purpose of the present studies was to determine the applicability of LAM to a range of tough problem areas in musculo-skeletal and dental research in which we are currently interested, and to find whether this approach also generated its own problems. We felt that it might overcome specific problems in the field of investigating the calcified tissues in bones and teeth and their junctions with adjacent soft tissues.

There are many different calcified tissue phases in bones (and teeth) and several types of junction between cells or a calcifiable or calcified matrix and a calcified tissue per $s e$. These junctions are often not hard and fast planes: to understand them, we need 3D information and they must suffer minimal disturbance during sample preparation. 
The approaches for correlative light and electron microscopy of hard tissues and hardsoft tissue interfaces which compete with LAM are:-

(1) to saw off the block face with a water-cooled diamond saw blade or band saw as in the Exakt system (Donath and Breuner 1982) after sticking it to a slide: then grinding and polishing a section (Gross and Strunz 1977; Plenk 1986; Wolf et al 1992; Erben 1997; Singhrao et al 2012). The disadvantage is the huge loss of tissue - typically 500 $\mu \mathrm{m}$ - per slicing event, which is impractical for most samples and impossible for small samples such as mouse bones or small surgical biopsy samples or rare tissue samples, and serial sectioning is impossible.

(2) confocal microscopic optical sectioning within a block surface. This is very productive approach that we have used extensively. It gives perfect correlation with SEM (Boyde et al. 2005), but transmitted light methods, including polarised light and staining, are not available.

(3) Serial block face imaging can be achieved by milling the top layer away, but this results in total sample destruction.

\section{The need not to decalcify.}

The structure and function of the mineralised tissues depends in part upon the nature, arrangement and concentration of the mineral component. Therefore, we must not remove the mineral component, which is a part of what we want to study. The classical approach to study hard tissues by light microscopy starts (after fixation) with decalcification, to make it possible to cut sections with a knife. This destroys the most highly mineralised tissues like dental enamel, and, very importantly, the high density mineralised infill (HDMI) material which we are discovering to be highly significant in understanding self-healing in dental tissues, early wound healing in bone 
microfractures and surgery, and as one mechanism of cartilage destruction in OA. Decalcified sections notoriously have the bugbears of separation between hard and soft tissues, crinkling, wrinkling and variations in section thickness. Undecalcified bone sections cut with a microtome are commonly employed, but examination of such preparations in 3D show that hard tissue structure and junctions with soft tissues are unmercifully destroyed and sections look like a ploughed field if studied in 3D. All these artefacts are avoided by NOT decalcifying and laser cutting.

\section{Materials and Methods}

All samples were archival and had been used in previous studies. All had been fixed in formaldehyde in neutral buffer for a period from one day to one week, stored in $70 \%$ ethanol until dehydration in $100 \%$ ethanol, substituted with xylene and then methyl methacrylate monomer, polymerised in situ to PMMA. Blocks were trimmed and finished by micro-milling or polishing to reveal planes of interest.

In every instance we had employed BSE SEM of topography free PMMA block surfaces. Most blocks were re-imaged using BSE SEM after iodine staining - wet, using a triiodide solution, or dry, by iodine vapour to reveal cells and uncalcified tissue like cartilage, tendon, ligament, osteoid and muscle ((Boyde 2012; Boyde et al., 2014a,b; Ley et al 2014). Many of the samples had also been studied by confocal fluorescence and reflection imaging (Boyde et al. 2005) and several by in-house highcontrast X-ray microtomography (XMT: Boyde et al. 2014a) or standard commercial microtomography ( $\mu$ CT: Boyde et al. 2017). 
Use of the LAM technique requires access to highly specialised equipment which is by no means widespread at present. We took a range of samples to the manufacturer's facility (Hannover, Germany) to prepare the sections.

\section{Rat femur prepared and donated by Rowiak}

We began by studying a sample prepared and kindly donated by the manufacturer (Dr Heiko Richter, LaserLab solutions $\mathrm{GmbH}$, Germany). Their primary aim in this area had been to produce sections which can be stained with a variety of normal histological and immunostaining procedures - as permanent mounts - and, as such, they remove the PMMA resin embedding medium prior to staining using a xylene substitute. This we wished to avoid at all costs because soft tissues with no embedding medium must shrink and collapse on drying before use in the SEM. We studied this sample, as received, with 3D LM methods.

\section{Equine bone}

Cracks in bone and calcified cartilage heal by the intercalation of a densely mineralised material - easily seen with BSE SEM - which is totally lost upon decalcification (Boyde, 2003). It was later discovered that this high density mineral infill (HDMI) material also extends into hyaline articular cartilage (HAC) as high density mineralized protrusions (HDMPs) which fragment to make a hard and sharp abrasive material within $\mathrm{HAC}$ - also lost to sight and mind after decalcification - and constitute one, as yet, hardly recognized method of joint destruction in osteoarthritis (OA). HDMPs were first clearly demonstrated in equine joints (Boyde et al. 2012; 
Ley et al. 2014; Laverty et al. 2015) and later found in human hip (Boyde et al. 2014a) and knee joints (unpublished).

\section{Thoroughbred McIII, Palmar Osteochondral Disease (POD)}

We prepared two distal third metacarpal condyle samples with advanced over-exercise related OA lesions selected from the Hong Kong study of palmar osteochondral disease (POD) in Thoroughbred racehorses (Boyde et al. 2011; Pinchbeck et al. 2013).

\section{$\underline{\text { Icelandic horse, OA hock joint, interosseous ligaments }}$}

Bones in the equine hock are tightly bound together by inter-osseous ligaments. We had samples from a study of OA in the Icelandic horse (Ley et al. 2014). We prepared one sample to isolate the region containing the central ligament attaching the third metatarsal to the third tarsal bone.

\section{$\underline{\text { Standardbred Carpal OA }}$}

One sample (of the type reported by Lacourt et al. 2012 and Laverty et al. 2015, being a $1 \mathrm{~cm}$ diameter cylindrical core) taken from the carpal bone of a Standardbred horse.

\section{Human bone}

\section{Anterior cortex of the lumbar vertebral body}

In a continuation of a study of changes in the lumbar spine with ageing and osteoporosis, we selected two of 50 fourth lumbar (L4) vertebral bodies (collected at UCL 1989-1993: Jayasinghe et al., 1993) and one second (L2) body from 70 from the 1992, qBSE study of the degree of mineralisation of 'bone' (European Union Concerted Action Biomed 1 "Assessment of bone quality in osteoporosis"). These 
were also studied by high contrast XMT imaging. We examined the anterior cortical segment, with annulus fibrosus and disc, after reducing the block surface area required for LAM preparation. Our key interest here lies in the incorporation of nonbone tissues into bone. Three samples selected were: 1) L4 Male 53y. 2) L4 Male 72y. 3) L2 Female 92y, iodine vapour stained.

\section{Hip OA, Alkaptonuria (AKU)}

One block from the femoral head of an AKU patient who had suffered severe pain from osteoarthritis, from the case described in Boyde et al (2014a). This sample had been imaged with XMT before embedding and again after the PMMA block preparation. We selected this as representative of the extreme difficulty in preparing sections of HDMPs.

\section{$\underline{\text { Knee OA, BML study }}$}

So-called bone marrow lesions (BML) are associated with OA and its pain (Kuttapitiya et al 2017). In a collaboration with Dr N. Sofat, St Georges Hospital Medical School London, we are studying some of this material. We prepared sections from one sample of distal femur. The problem here is certainly not the shortage of tissue, since we produce large numbers of sub-blocks from the material obtained at one arthroplasty. It is rather focussing on specific regions to obtain quality data.

\section{Fibrogenesis imperfecta ossium (FIO)}

FIO is an exceedingly rare bone disease. We used one small sample from a 50 year old male trans-iliac core sample donated by Dr R. Smith, Oxford, from the case reported in Carr et al. (1995). 


\section{Dental implant site bone core}

Small bone samples can be recovered from the trephine drills used to prepare sites within jaw bone at which implants are to be placed. Evaluation of bone quality in such surgical waste biopsies may prove to be of value in future research in implant dentistry. We chose one sample at random from the series collected by Wong (2000). Such cores are 2 or $3 \mathrm{~mm}$ diameter and in some cases rather fragmentary if the bone quality was poor. It is difficult to conceive that any other technology could be employed to produce more than one undecalcified section for LM and SEM.

\section{Mouse bone}

Mice are studied intensively to contribute to understanding basic mechanisms in bone physiology and pathology. As regards the use of undecalcified, intact tissue sections (i.e., not microtomed), PMMA blocks have been used extensively to acquire electronoptical sections for qBSE-SEM analysis to study mineral concentration and optical sections by fluorescence CSLM to study histology and dynamic histomorphometry from the interpretation of calcein or tetracycline double labels (e.g., Bassett et al. 2012a,b).

We chose three types of mouse bone sample: a) LS caudal vertebrae: b) LS distal femur and proximal tibia: c) TS proximal tibia.

\section{$\underline{\text { LS tail vertebrae. }}$}

Nod Scid mouse tumour invasion model, caudal vertebra. 13-week-old female nude (NOD SCID) mouse bearing metastases of subcutaneous breast tumour. Sham 
(vehicle only) treated sample (donated by Philippe Clézardin, Lyon: Fournier et al., 2010). We had shown excellent histology of the tumour spread within caudal vertebrae after iodine staining BSE SEM (unpublished).

\section{$\underline{\text { LS distal femur and proximal tibia. }}$}

Phospho1 knockout mouse. The phosphatase PHOSPHO1 is involved in the initiation of biomineralisation. For 3D characterisation, we used X-ray micro-tomography. SEM disclosed defective mineralising fronts and extensive patchy osteomalacia, which had not been previously recognised (Boyde et al. 2017).

\section{$\underline{\text { TS proximal tibia. }}$}

E11/Pdpn conditional knockout mouse. The transmembrane glycoprotein E11/Podoplanin (Pdpn) is involved in osteocyte differentiation and dendrite formation in vitro (Zhang et al. 2006; Staines et al. 2016). Staines et al. (2017) generated a bonespecific conditional Pdpn hypomorphic mouse and confirmed a role for Pdpn in the attainment of fully elongated osteocytic canalicular processes. We conducted extensive studies using high resolution fluorescence CSLM to study detail of the mineralising fronts and rates of bone apposition in TSs of the proximal tibia, as well as iodine stained BSE SEM, and we selected one of these for study.

\section{Human dental enamel}

Tooth enamel is the hardest mammalian tissue, being composed of highly oriented carbonated-hydroxyapatite crystal bundles to typically $91 \%$ by volume, $98 \%$ by weight (Boyde, 1989). It has a strong disposition to cleave parallel with its prism boundary discontinuities and incremental layer lines, and, consequently, it is 
extremely difficult to prepare as unbroken, undistorted thin sections for LM studies. There are several reasons why we would like to have sections with thicknesses approximating to the average cross sectional dimension of the individual enamel prisms. We studied one PMMA embedded human third permanent molar, LS in block surface.

\section{Laser ablation microtomy /machining (LAM) a thin section from a block face}

The necessary 'Tissue Surgeon’ equipment from LLS Rowiak LaserLabSolutions $\mathrm{GmbH}$ enables us to produce thin, plane-parallel histological sections of resinembedded tissues. It works by scanning a femtosecond pulsed $1030 \mathrm{~nm}$ laser through a cutting plane in the specimen. Laser energy is focussed by a high numerical aperture objective lens into a very small specimen volume and for an extremely short period, generating a high instantaneous laser flux. A plasma ball is produced that obliterates the specimen only at the focal spot. Little specimen damage occurs outside the focal spot, because the absolute amount of energy deposited in the specimen is low, and spread out where the laser is unfocussed. Continuous ablation within the cutting plane results in separation of the section from the parent block. With this method, the block face and the underlying few microns comprise the section after laser cutting.

- The block face is glued to a standard glass light microscope slide with a cyanoacrylate adhesive (Panzer Kleber http://www.panzerkleber.de/produkte/).

- The slide with the attached block is placed on the equipment.

- It is examined by an inbuilt optical coherence tomography imaging system. We focus through the glass slide, the cyanoacrylate adhesive, the block face, and a further increment into the specimen to predetermine the section 
thickness. We also level the slide so that the resultant section will have uniform thickness.

- The focussed, pulsed laser beam is scanned along a $1 \mathrm{~mm}$ line, and this swathe is translated in an XY scan to cover the entire area of the block, which is thereby released from the slide, leaving the section stuck to the slide.

- To appreciate the LAM cutting damage, both the blocks and the sections were studied by SEM in the as-machined condition. The as-cut surfaces were not useful for obtaining SEM based results.

- The main aim is achieved after minimal water lubricated polishing with 4000 grit 'wet and dry' silicon carbide paper to remove 1-2 $\mu \mathrm{m}$ of cutting relief from the surface of the section.

- The section attached to the glass slide is washed with distilled water and blotted dry. We avoided the use of ethanol.

- For SEM, we examined the slide-mounted, uncoated sections by BSE imaging (Zeiss EVO MA10, Cambridge UK), usually at 20kV accelerating voltage, 50Pa chamber pressure, working distance 6 to $10 \mathrm{~mm}$ [NB the 4 sector BSE detector is within this distance], and beam current 0.5 to $1 \mathrm{nA}$.

- We went backwards and forwards between SEM and LM studies.

- For LM, we applied a coverslip using glycerol: this is easily washed off with water and does not attack PMMA or cyanoacrylate.

- Polarised light microscopy is possibly best done before staining.

- Many water soluble stains work with the PMMA left in place. We have used Toluidine blue, Orcein, MacNeal's Tetrachrome, Iodine Green, Acridine red and Victoria blue. 
- For correlative studies using LM and SEM, either set of images may be recorded first. It is very convenient if the LM image can be displayed on a monitor next to the SEM.

- For correlative studies with XMT, it is best if the PMMA block is studied by $\mathrm{XMT}$ just before the first section is removed from the block face, since this plane will be automatically found in the reconstructed 3D XMT data set and will register perfectly with SEM and LM images of this section.

- Images from different types of microscope were merged using home made software ('Honza's' programme, Boyde et al. 2005, available free from the author).

\section{Results}

\section{Rat femur LS LAM LM section prepared by Rowiak}

The 3D LM methods we used to study this section were (1) production of through focus image series which were processed to produce stereo-pairs using AutoMontage software (Syncroscopy, Cambridge, UK: unfortunately this firm is no longer marketing copies of this software): and (2) in house dynamic aperture microscopy (DAM) whereby we rotate a 90 degree open sector stop in a specially constructed condenser to obtain multiple oblique views, which, when played in sequence, give a fully $3 \mathrm{D}$ view of the sample. They showed that the soft tissue regions in the sample, such as the bone marrow and the periosteum, had shrunk back relative to both mineralised bone (Figure 1), but also relative to osteoid where present. We also found that the staining of cells within mineralised bone was not uniform in depth. 


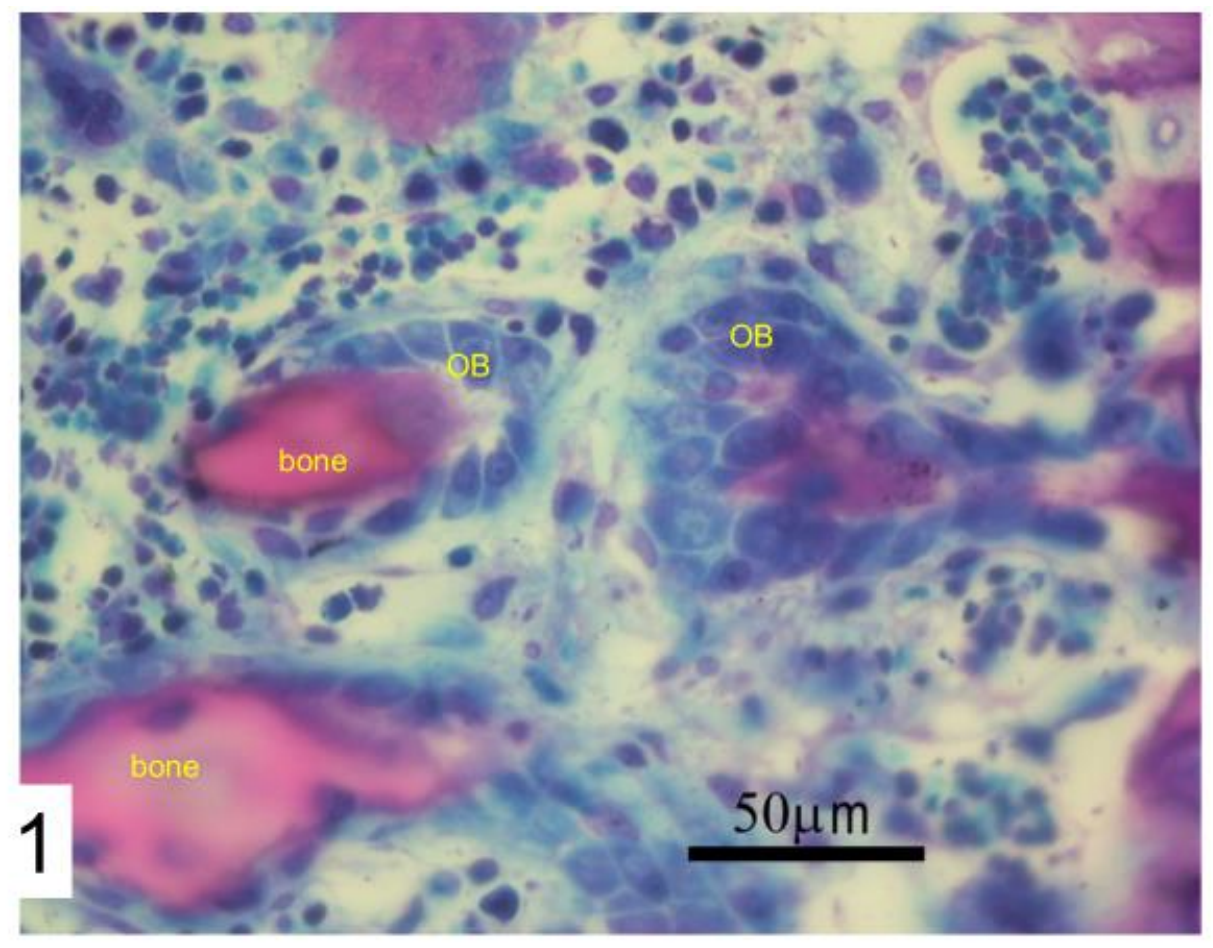

Figure 1. LM, 10 $\mu \mathrm{m}$ LAM section of rat femur, 'deplastified' in Shandon Xylene Substitute (https://www.thermofisher.com/order/catalog/product/6764506), Sanderson's rapid bone stain and Van Gieson counterstain. Focussed at level containing bone marrow and osteoblasts (blue). Mineralised bone (pink-purple) is out of focus because it hardly shrinks due to its high collagen and mineral content. Preparation donated by Dr Heiko Richter. [In the rest of the study reported here we have left the PMMA in place to prevent such shrinkage].

\section{General observations re the LAM method for correlated LM-SEM}

Satisfactory results were obtained with tissues from all the samples selected.

However, BSE SEM examination at the as-cut stage of all the blocks and the slides showed the pattern of the cutting sequence, so that the history of the movement of the laser focus could be traced in detail (Figure 2A,B). Thus every movement of the cutting beam left a curved, linear trace in the surface, and the overlap between the millimeter wide cutting swatches could be easily seen: indeed these edges were where 
undesirable damage was most frequent. It sometimes appeared as if alternate strips in which the $\mathrm{Y}$ tracking direction is reversed - were machined differently and to different depths (Figure 2B). We speculate that this might be related to a hysteresis affect in the mechanical scanning mechanism such that the set depth is different in alternate scan directions. If this is the case, then one solution would be to blank the beam on return and scan only in one Y direction having first determined which is the 'good or better' direction.
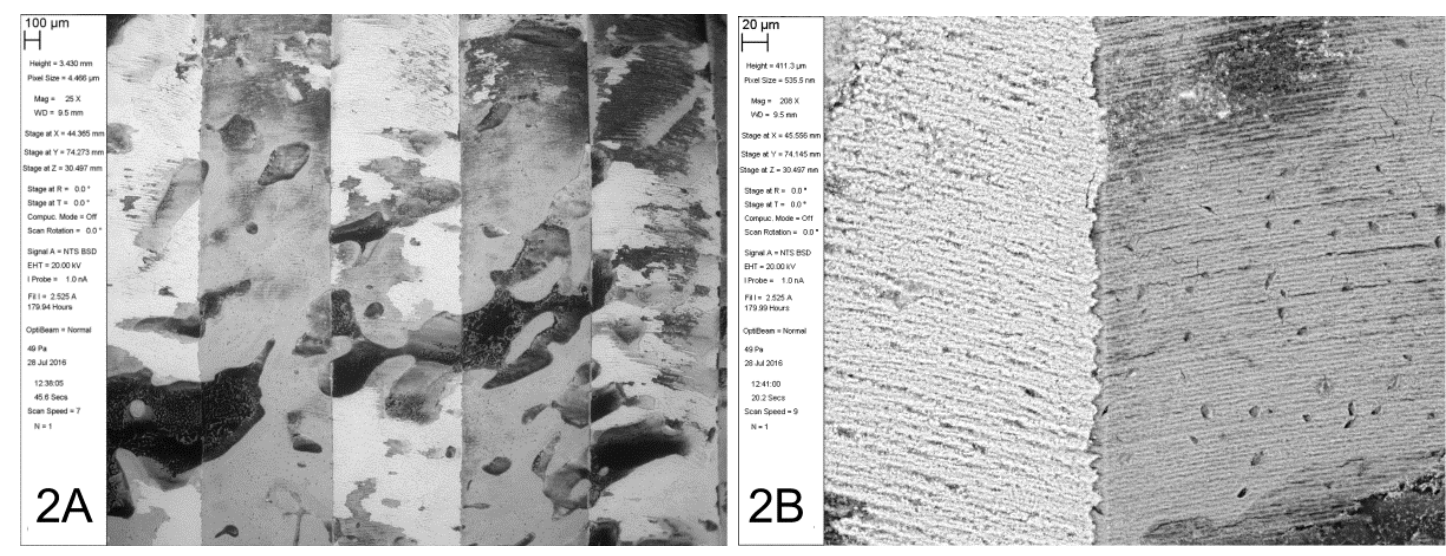

Figures 2A \& B. Subchondral trabecular bone in TB racehorse distal third metacarpal condyle. 20kV BSE SEM of LAM section on glass slide, as removed from machine, uncoated $50 \mathrm{~Pa}$ chamber vacuum. A. Alternate $1 \mathrm{~mm}$ wide strips (swathes) in $\mathrm{Y}$ direction have a different surface texture, accounting for the difference in overall brightness seen here. C372lf slide1_017. B. Shows border between two swathes in solid bone. Note the individual cut lines in $\mathrm{X}$ direction are curved. Texture of the cut surface is smoother in the darker band to the right.

In some instances, tissue was removed almost in its entirety where the section thickness chosen was too small and/or where the levelling process was unsatisfactory. In some cases - early in the learning process - cyanoacrylate adhesive and tissue had been burnt black by heating where the laser focus was within the glass slide, but the 
resultant damage was to material on the slide, not to the parent block, so that satisfactory sections were obtained after resurfacing the block before the next attempt.

PMMA blocks may warp with prolonged storage, which we have presumed to be due to the loss of firmly-bound, structural water in tissues which is not removed by ethanolic dehydration prior to infiltration with methacrylate monomer, but this water can eventually leave a polished surface in a dry atmosphere. It was observed that the better results were obtained when taking a second (or later) section, after on-the-spot re-flattening and re-polishing of the block immediately before it was stuck to the slide with cyanoacrylate.

The bond between cyanoacrylate and PMMA embedded hard and soft tissues rarely failed at any stage in sample handling. One slide was broken after the polishing step: viewing the resulting fragments on edge confirmed the good bond from tissue to glass via the super-glue (Figure 3).

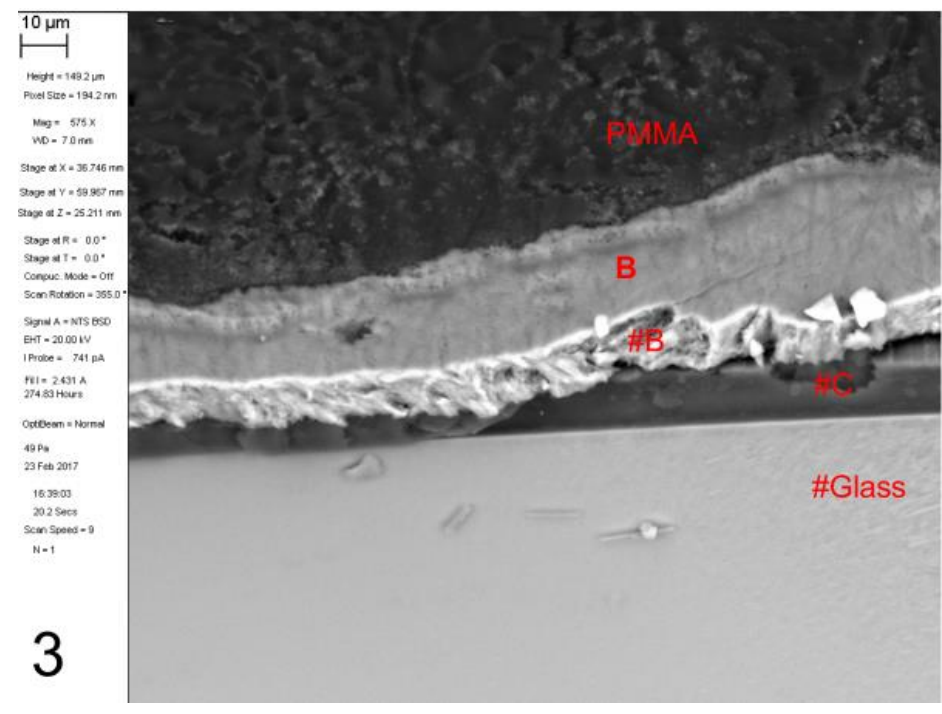

Figure 3. Subchondral trabecular bone in human distal femur, after accidental breakage of slide. Fragment mounted with 45 degree tilt such that section tilts 45 to 
the top and fracture surfaces $\sim 45$ to the bottom. $\# \mathrm{~B}=$ broken bone. $\# \mathrm{C}=$ fractured cyanoacrylate film; $\# \mathrm{G}$ = fractured glass; $\mathrm{B}=$ Bone; $\mathrm{P}=$ PMMA containing marrow.

\section{Equine bone}

POD in Thoroughbreds.

We studied two distal third metacarpal condyle samples with advanced OA lesions selected from the study of palmar osteochondral disease (POD) reported in Boyde et al. (2011) and Pinchbeck et al. (2013) (Figures 4 \& 5). In both cases, HAC had been entirely missing over parts of the most severely affected - the most indented or depressed regions. In other words, they both showed the most severe OA grade. Both samples had been documented by photography immediately on opening the joint, and again after cutting antero-posterior slices of the distal palmar condyles; by Faxitron point projection digital x-ray microradiography; after embedding and block preparation by qBSE SEM, CLSM and again by BSE SEM after iodine staining. The LAM sections were montaged by BSE SEM (Fig 4A) and PLM (Fig 4B) and LM after Tetrachrome staining (not shown). Detail in PLM and SEM images is obviously complementary.

Detail in Figure 4 shows a large fragment of ACCs surrounded and included by new bone which has formed around it, towards the remade joint surface. Within this, the typical morphology of an ACC MF can be identified - but cartilage which must have been $\mathrm{HAC}$ at the time that the fragment originally broke away has mineralised. 

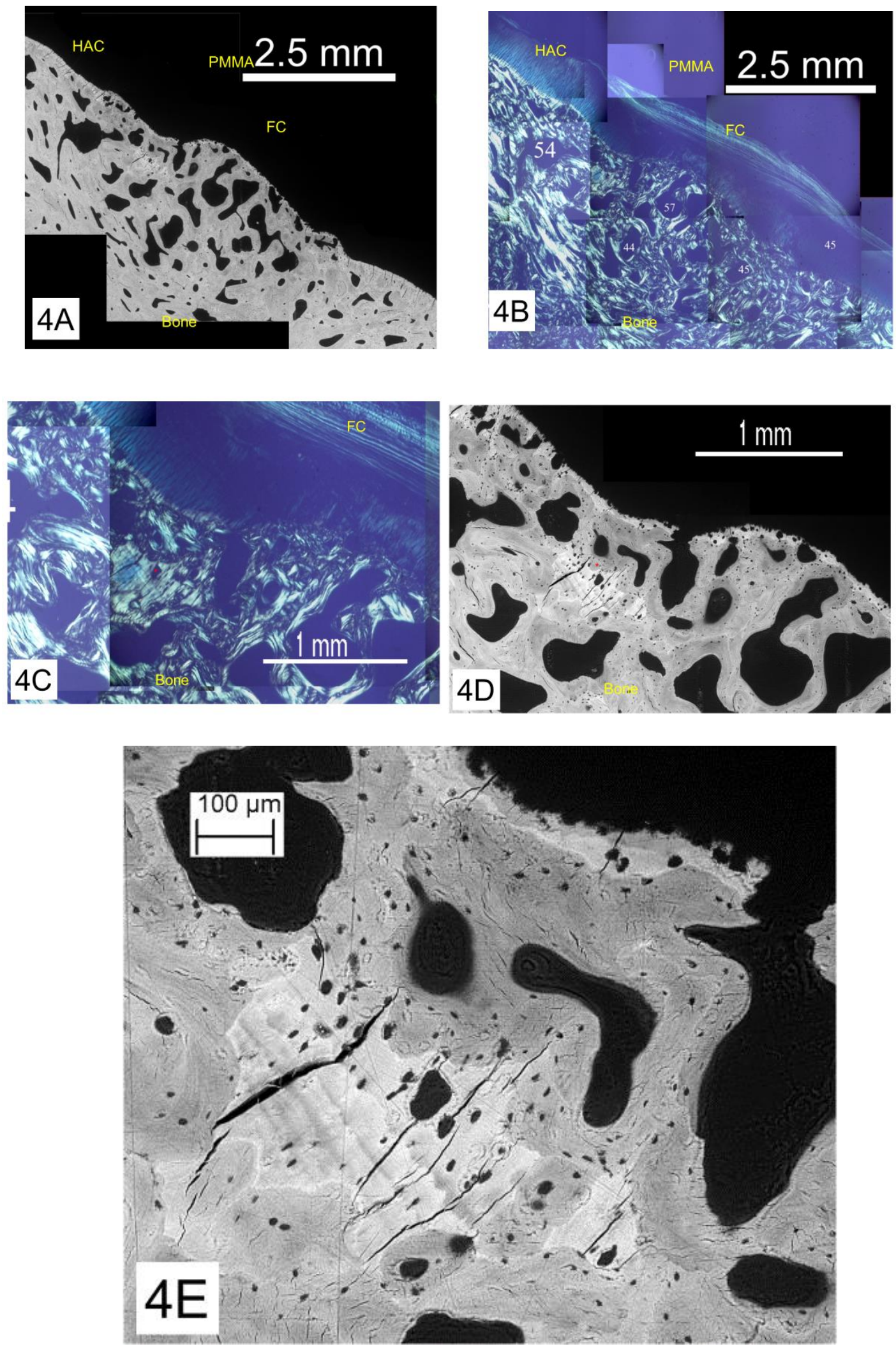

Figure 4 A-E. TB racehorse distal third metacarpal condyle, case with severe grade OA (POD). A. Low power BSE SEM montage. B. Linear PLM of same section, same region, showing collagen orientation in SCB, ACC and HAC: FC = fibro- 
cartilaginous superficial layer with fibre orientation parallel to joint surface. $\mathbf{C}$.

Higher magnification within same montage. D. Identical field in BSE SEM: E.

Centred on an isolated patch of the original ACC which had previously broken away and is now included in bone formed deep to a new ACC MF. The original ACC MF lies within this patch: tissue above this line is also calcified and may have calcified after the fragment was formed. The entire fragment is surrounded by scalloped border indicating prior osteoclastic resorption.

In the second case, Figure 5A-C, we see an area outwith the main depressed area of the OA lesion, at an edge where already thinned HAC is being lost from the ACC MF.
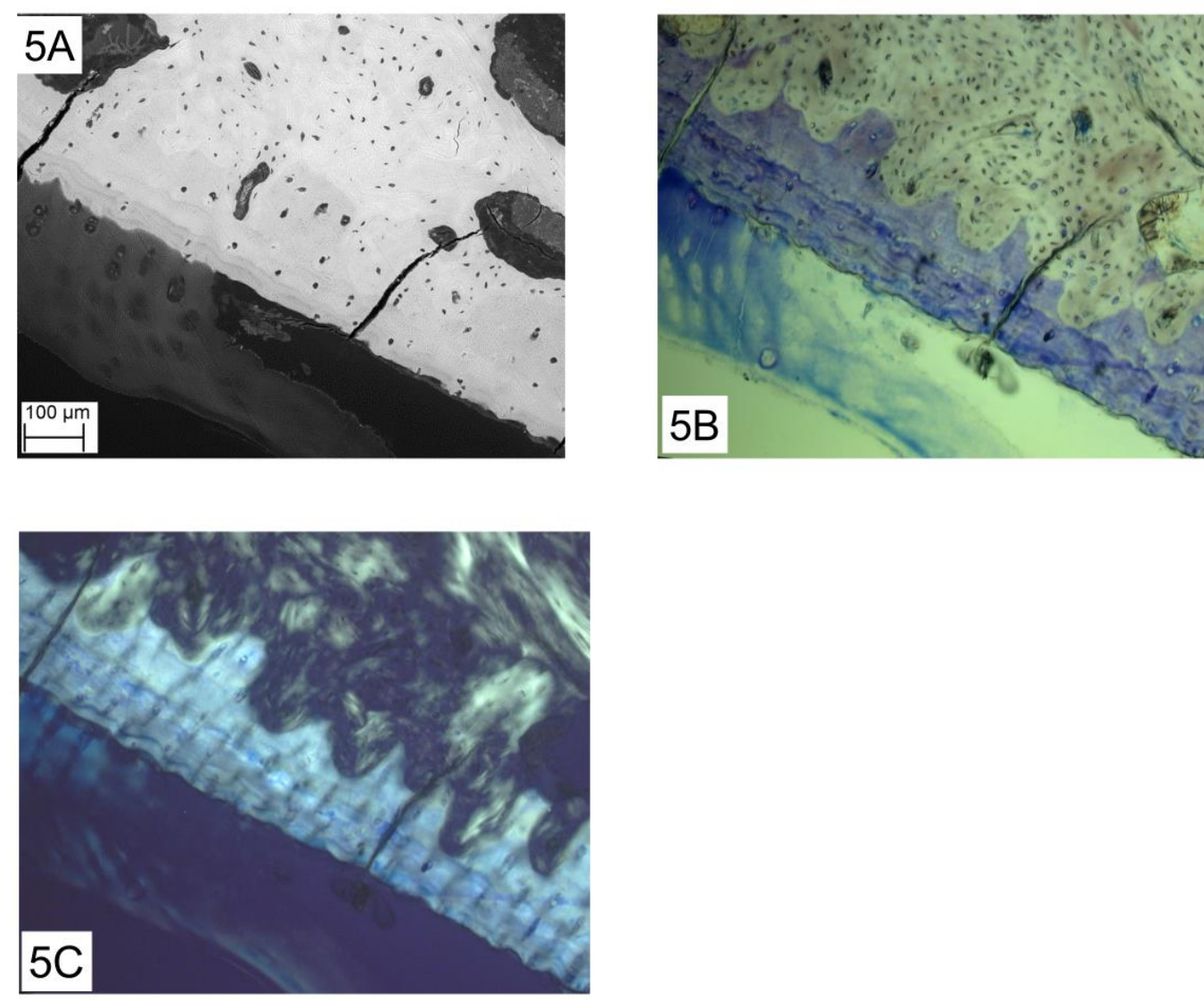

Figure 5. TB racehorse distal third metacarpal condyle, another case with severe grade OA (POD). Same field of same LAM section: A. BSE SEM B. Tetrachrome staining. C. Linear PLM. Separation of HAC from ACC was shown in every imaging modality. 


\section{$\underline{\text { Icelandic horse MT3-T3 joint }}$}

Here, we examined two bones joined together by extensive interosseous ligaments. The sample was cut from a much larger block with CT-T3-MT3 in a row (Ley et al., 2014). It contained the central T3-MT3 fossa together with surrounding, opposing HAC joint surfaces. Images showed excellent correlation between LM, PLM and BSE SEM (not illustrated).

\section{Carpal OA in Standardbred horses.}

Standardbred horses develop overuse OA in their carpal bones (Lacourt et al. 2012) and we had previously shown that this was associated with HDMI and HDMPs (Laverty et al. 2015). The case that we studied showed a high grade of OA, with a depressed region in the articular surface core sample, deep to which was a patch of newly formed cartilage, both uncalcified and calcified. The linear crack in ACC shown in Figure $6 \mathrm{~A} \& \mathrm{~B}$ extended for some distance perpendicular to the plane of this section, since it was seen in the parent block and in more than one section plane. This crack contains HDMI which is seen clearly in the BSE SEM image, but this could not be discriminated in either the PLM or the stained LM images. This is a surprising finding. It is obvious that HDMI (and HDMP) material will disappear in decalcified material, and this is at the heart of why these phases went undiscovered until BSE SEM came on the scene (Boyde 2003; Boyde et al. 2011). It is, however, not obvious that they should escape detection in the LM, even when we can show them and know they are there. 


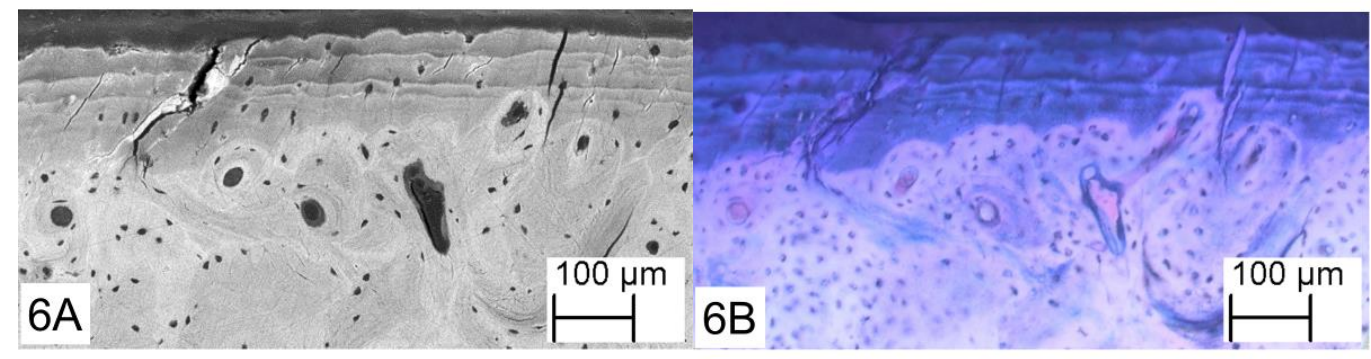

Figure 6A \& B. Standardbred racehorse carpal bone from a case with moderate grade OA. A. BSE SEM. B. Stained with Victoria Blue and Acridine red. The HDMI in ACC is prominent in the SEM image but cannot be identified as different by LM. It is interesting that the occurrence of HDMI and HDMPs in this material was first detected by re-examination or archival x-ray microtomographic data (Laverty et al. 2014). It had been confirmed in this autopsy core sample by SEM of both a macerated half and this PMMA embedded half.

\section{Human bone}

$\underline{\text { Structure of the cortex of the human lumbar vertebral body. }}$

We have been involved in an extended and extensive study of the degree of mineralisation of 'bone' in lumbar vertebral bodies (EU pre-brexit Action Biomed 1 Assessment of bone quality in osteoporosis). In the qBSE phase of this study, we found a wide range of mineralisation densities in 'bone', and of particular relevance in the present context, layers of higher density tissue at the anterior cortical boundary. Later, study of this same region via iodine staining showed that these strips were calcified ligament (or Sharpey fibre bone or dense fibrous periosteum). We needed a method to sort out the histology, and we believe that LAM provides it. We have excellent correlation between XMT, BSE-SEM, iodine vapour stained BSE SEM, and - in LAM sections from three individuals - SEM, LM staining and PLM (Figure 7A- 
E). Sharpey fibre bone, calcified ligament, calcified fibrous periosteum and cortical bone per se cannot be distinguished by x-ray microtomography.
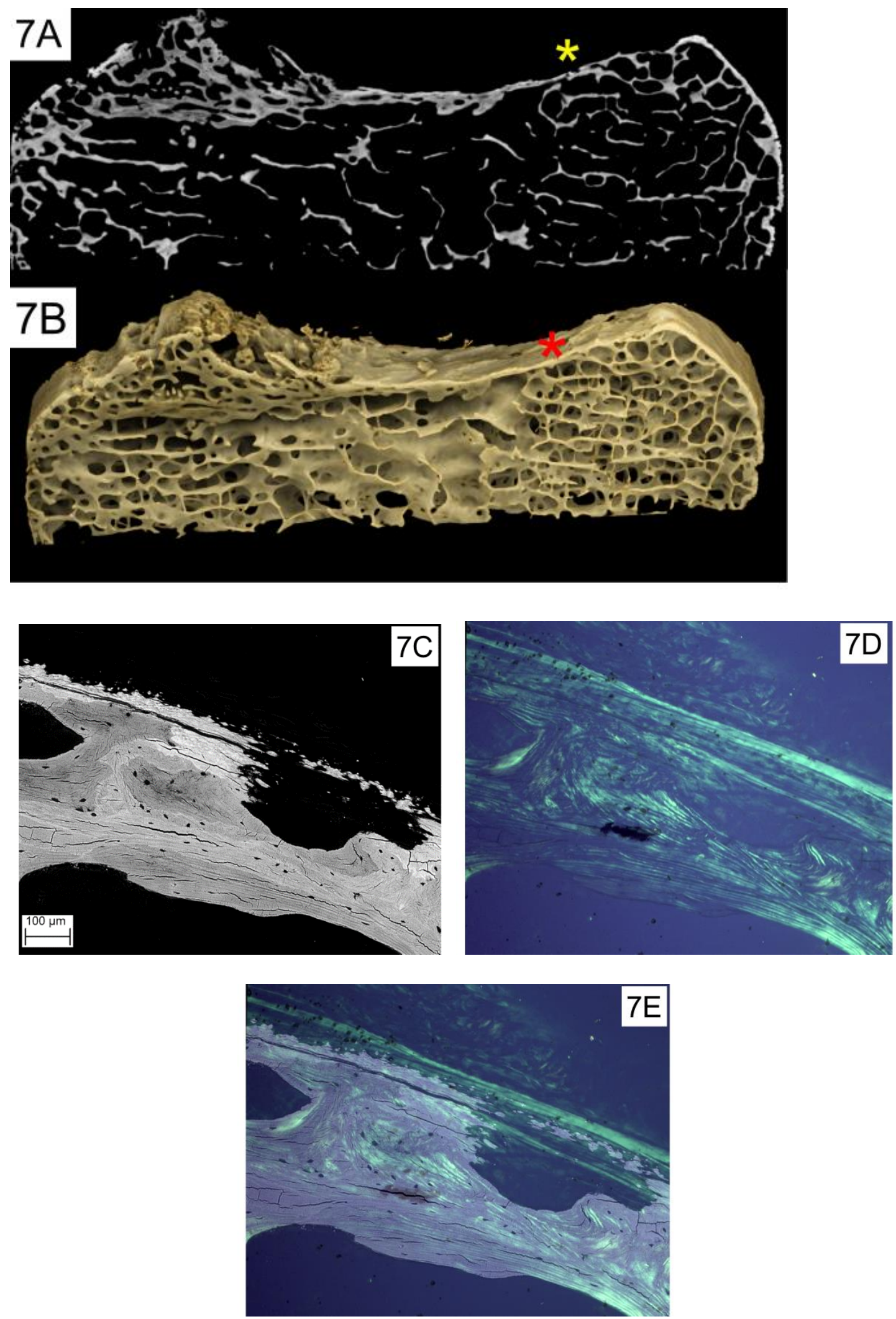
Figure 7A-E. Anterior portion of vertical slice through human fourth lumbar vertebral body, male 53 years old. A. single high contrast XMT slice near 'front' of PMMA block which is $30.5 \mathrm{~mm}$ wide. B. Drishti 3D rendering of XMT (both A and B are mirrored to present the same aspect as the following which are taken from the LAM slide). C. BSE SEM. D. PLM. E. Mixed SEM and PLM. Asterixes (*) in A and B show location of field in C-E. Parts of the anterior cortex originate as - and in ligament. This mineralises at or near the contact surface with calcified bone. Note that mineralised ligament cannot be distinguished from the non-mineralised portions in the PLM image. [Deeper parts of calcified ligament will be resorbed by osteoclasts and replaced (remodelled) by bone].

HDMPs in Human Alkaptonuria (AKU) related OA.

The extensive HDMPs in this case had originally been discovered by high contrast XMT of the whole femoral head, then retro-discovered in an MRI data set, and this knowledge used in preparing macro-slices for PMMA embedding, and the eventual demonstration by BSE SEM (Boyde et al. 2014b). This is a classic case where the 'baby would have been thrown out with the bath water' in the decalcifying fluid - if we had gone that route. The HDMPs could be sectioned by LAM, as shown in Figure 8A\&B. 

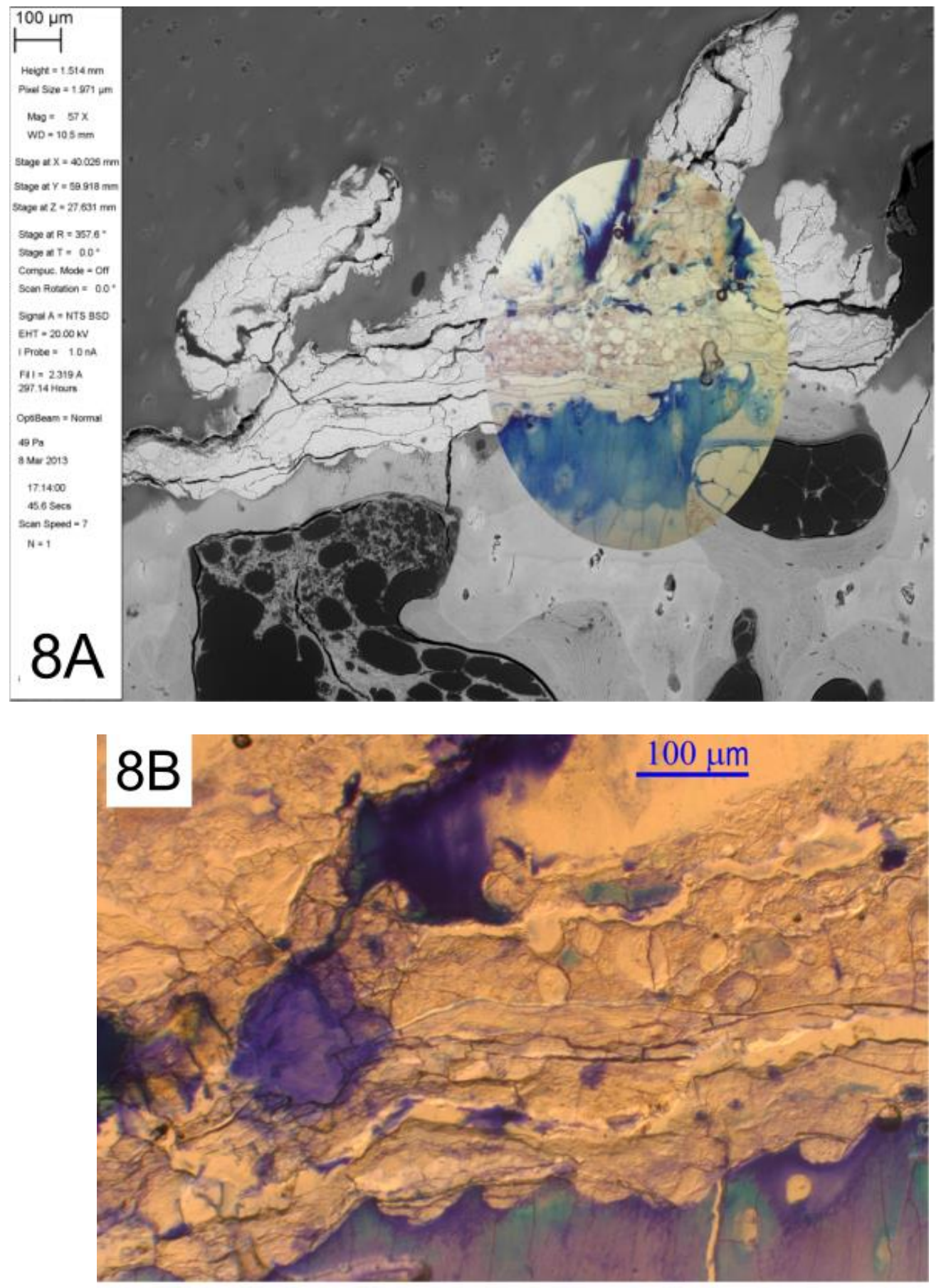

Figure 8. Femoral head with prominent HDMPs from AKU case presenting with severe OA pain, necessitating the arthroplasty: case presented in Boyde et al. 2014a). A. Background = BSE SEM of block surface after staining with ammonium triiodide. Note iodine staining of HAC matrix and soft tissues in bone marrow. Oval inset 
region $=$ LM of LAM section stained with Tetrachrome. B. Higher magnification of HDMP material [LM images are here mirrored].

\section{Human knee OA.}

Here we looked for - and found - OA findings we have previously made by SEM in both equine and human $\mathrm{OA}$, including changes in HAC histology, trabecular excrescences (Taylor et al. 2012) and some HDMI (Boyde 2003; Boyde et al. 2014a).

Figures 9A-C show how small hyperdense patches in ACC can be easily seen in the BSE SEM image, but cannot be detected in either the PLM or stained LM images.
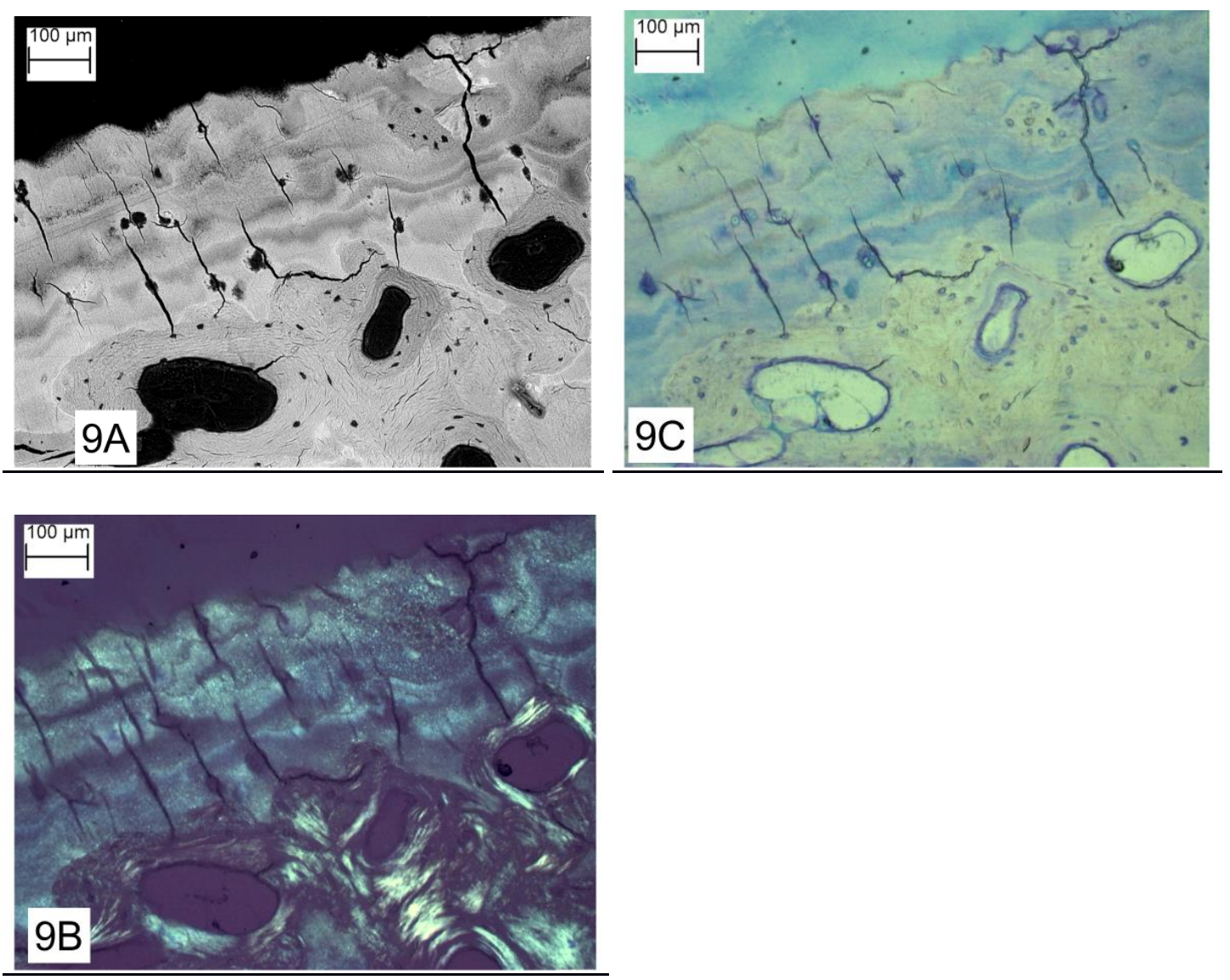

Figure 9A-C. Knee OA arthroplasty. Distal femoral joint surface. LAM section. A:

BSE SEM. B. PLM. C: Tetrachrome stain. Small high density (whitest) patches seen in SEM show no distinguishing features in LM or PLM. 
Fibrogenesis imperfecta ossium.

FIO is an exceedingly rare bone disease (Carr et al. 1995). The problem was how to get a section of very poor quality bone and osteoid from an archival PMMA block. We know of no other method with which the small amount of tissue could be resectioned to produce even one undeformed, undecalcified sub-sample without destroying most of the material. We confirm the known findings re FIO - that there is a large proportion of osteoid to bone and that the PLM image of both is weird, with only limited regions of birefringent lamellae. Figure 10 is a composite image of the LAM slide with BSE SEM, LM Tetrachrome and PLM components, the latter being added in as the negative component so that birefringent lamellae appear dark brown against the grey mineralised bone. Osteoid appear as pale blue-green.

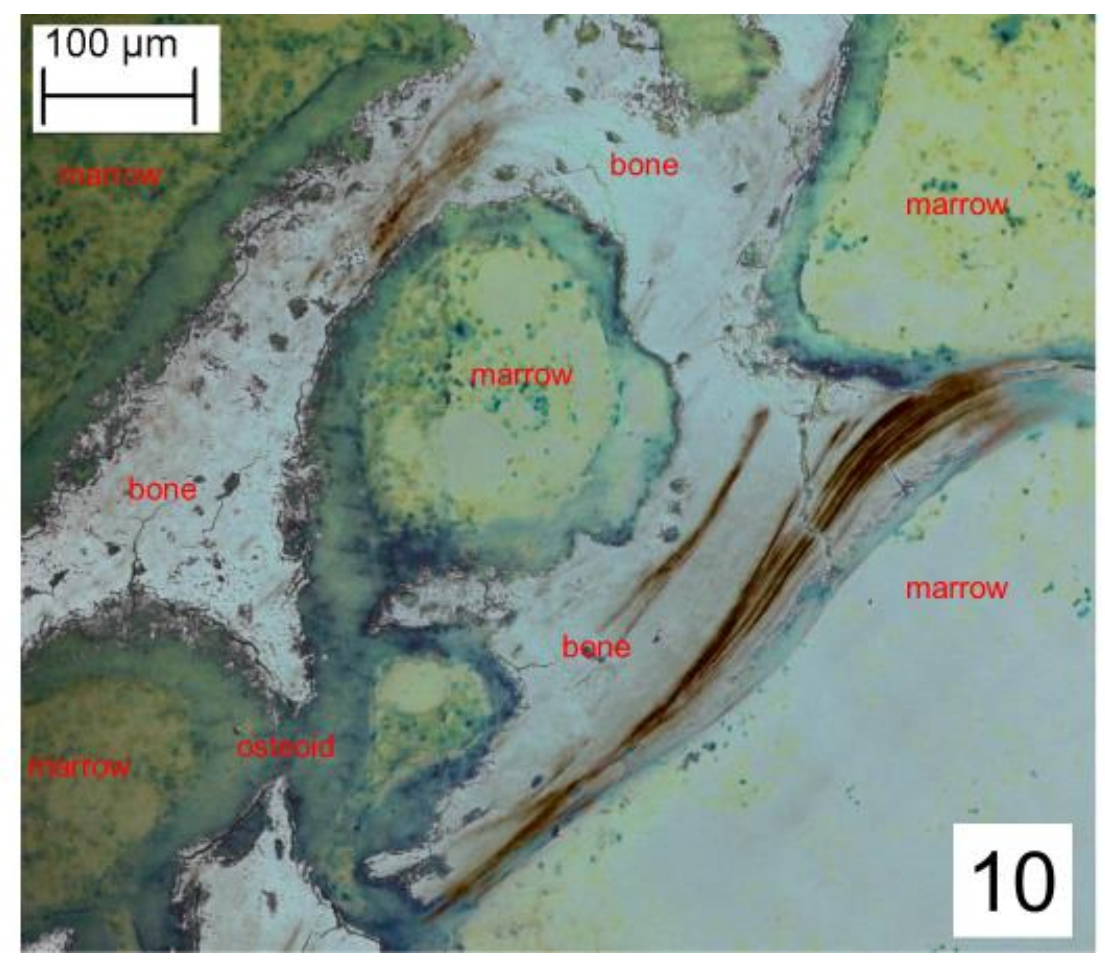

Figure 10. Fibrogenesis imperfecta ossium. Iliac crest. Combined BSE SEM, PLM and stained LM. To contrast patchy, bright birefringent lamellae seen in PLM with grey BSE SEM, negative version of PLM was used so that they appear as dark brown. Osteoid blue-green. 


\section{Dental implant site bone cores.}

The cores from trephine drills used to prepare a dental implant site are amongst the tiniest bone samples we handle (Wong 2000). Pieces of bone broken away in the drilling operation are impacted into the periphery of the sample such that only very narrow regions in its centre are tolerably intact. In addition, many core samples are discontinuous transversely - unless the bone was very dense. Nevertheless, we were able to find intact regions of thin sections of the centre of the core showing good quality lamellar bone (Figure 11).

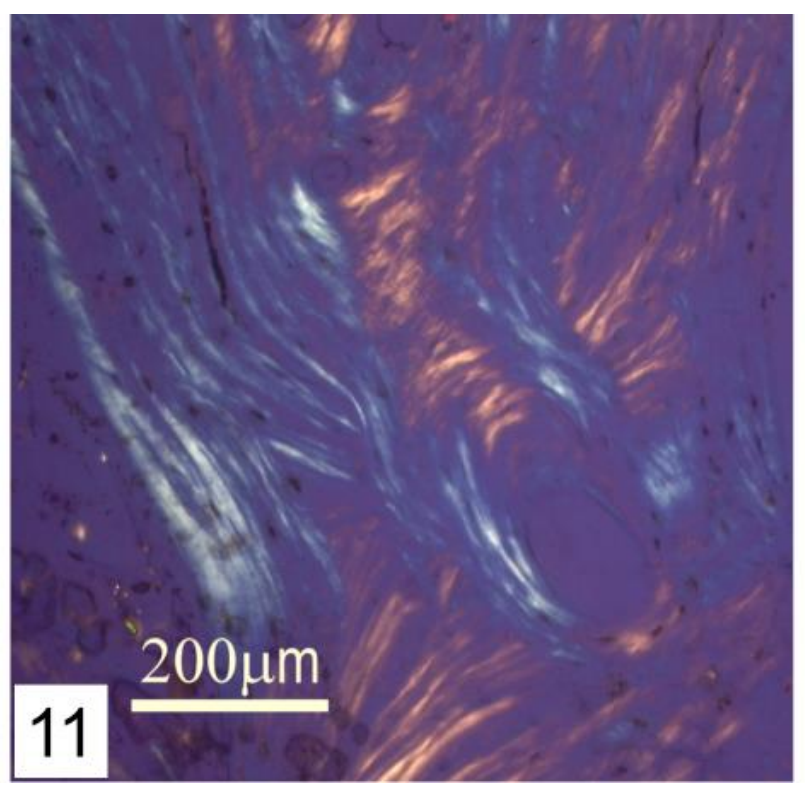

Figure 11. Dental implant site core, human mandible, surgical scrap. Lamellar bone within trabeculum in centre of core. PLM with half wave plate: collagen orientations in one sector of the erstwhile Maltese cross are yellow, and in the other, blue.

\section{Mouse bone}

$\underline{\text { LS tail. }}$

Breast cancer tumour invasion model in nude (Nod Scid) mice. LSs of a chain of mouse caudal vertebrae are samples we very frequently encounter. With respect to PMMA embedded material, the usual problem is to assess the bone mineral density 
from two perspectives: 1) the volumetric density of bone tissue, for which the BSE

SEM image is ideal for stereology because of the thinness of the electron optical section, such that it satisfies stereological rule no. 1 - that the sample be infinitely thin - is satisfied, whereas this is not true for LM sections: and 2) the bone fabric density by qBSE (Boyde et al. 1999) - the relative mineral concentration at a sub-cubicmicron resolution scale - far better resolution than obtainable by $\mathrm{x}$-ray microtomography.

LAM results from the sample studied would make no contribution to studies of tumour metastasis within the bone organ - since here decalcified section histology provides all the excellent detail that is needed for the problem at hand, and the PMMA-LAM approach is vastly more time consuming. However, it did show that we could prepare continuous undeformed sections of a row of vertebrae and intervertebral joints. We illustrate this with a PLM image showing the orientation of the fibre bundles in the annulus fibrosus (Figure 12).

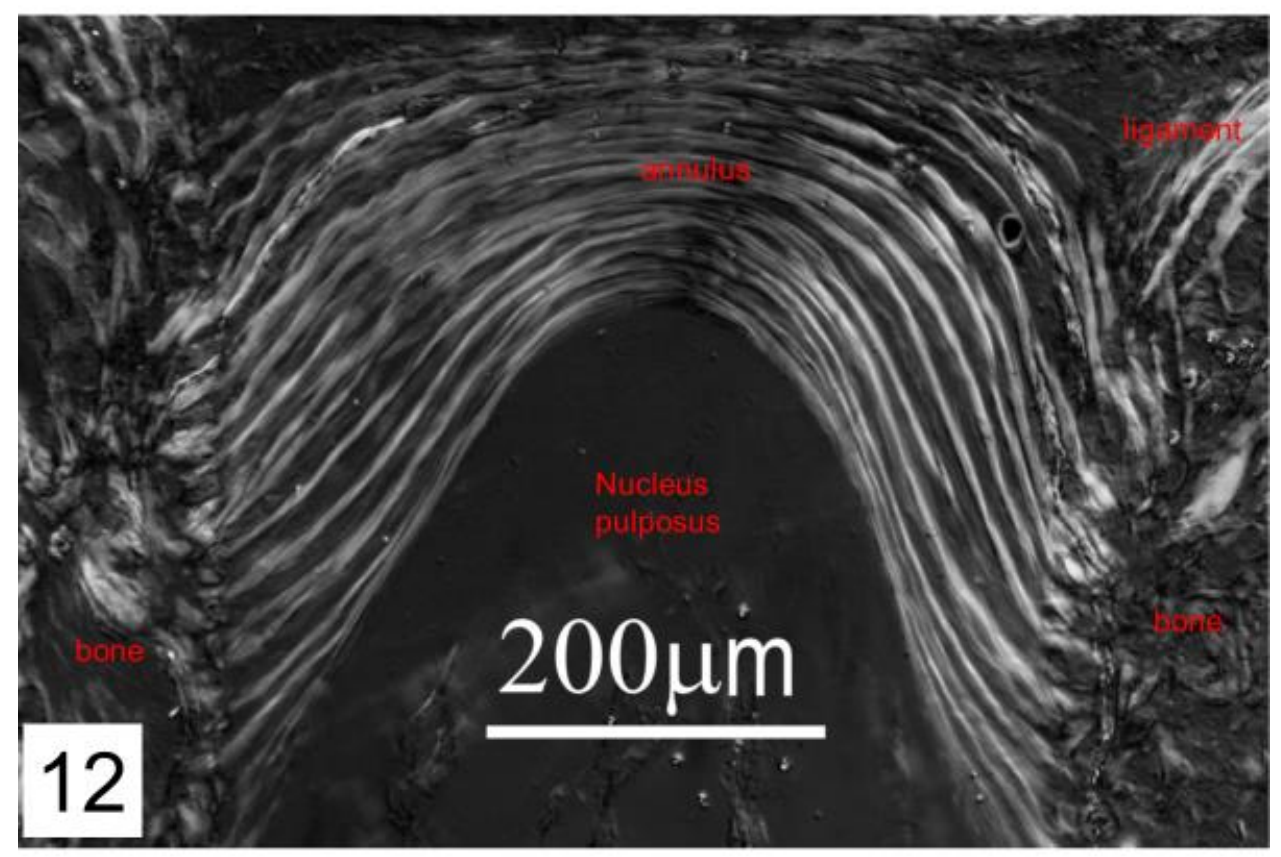


Figure 12. Annulus fibrosus of intervertebral joint in mouse tail. To minimise the effect of in plane orientation on the brightness of the birefringent layers and maximise the contrast, PLM images were recorded at three 30 degree rotations of crossed polars. Then a derivative darkest-of-all-three was subtracted from lightest-of-all-three. As with all LAM sections, calcified tissues and non-calcified regions are undistorted: no wrinkling or crinkling.

\section{LS knee.}

Phospho1 knockout mouse. LSs of femur and proximal tibia cut in a parasagittal plane are very common in our work with mouse genetic models. We have previously reported on the combined XMT, PMMA-BSE-SEM, macerated 3D SEM sample study of this material (Boyde et al. 2017). Extensive regions of both compact cortical and trabecular bone matrix in Phospho1 KO mice contained no significant mineral or showed arrested mineralisation fronts, as failure of fusion of the separate mineralising micro-volumes within bone. Figure 13 shows the border between mineralised bone (red) and a large osteoid patch (paler, bluer) in a Tetrachrome and acridine red stained

\section{LAM LM LS.}




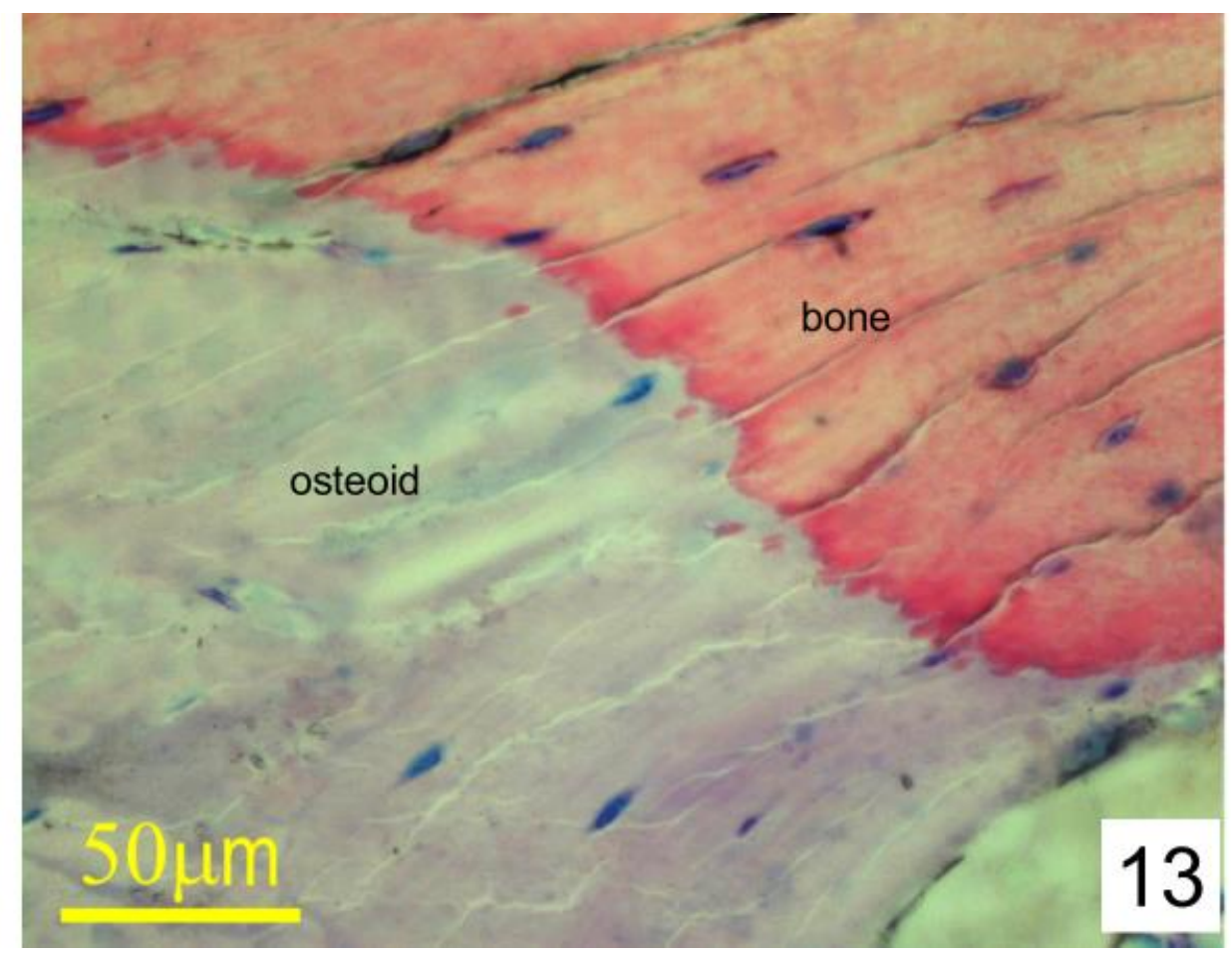

Figure 13. Subperiosteal bone in proximal tibial cortex of Phopho1 knockout mouse. Stained Tetrachrome and acridine red. Light blue $=$ unmineralised bone matrix (osteoid). Red $=$ mineralised bone. Osteocytes in both bone and osteoid are darker blue.

\section{TS tibia.}

E11 knockout mouse. Researchers studying Mineral Apposition Rate (MAR) by calcein double labelling of small rodent long bones have used microtomed longitudinal sections (LSs) of PMMA embedded tissue, with serious disadvantages. First, microtomed, undecalcified bone is always badly deformed, although the rough position of intra-vital labels is retained. Second, LSs are the least satisfactory viewpoint for studying bone growth and drift. This is because cortical bone predominantly forms endosteally - burying trabeculae within it - in the tapering ends of long bones. Furthermore, trabeculae themselves 'drift' in this plane. A far better 3D understanding results from studying transverse section (TS) series, and the sample we 
used here came from our study using this approach. The TS proximal tibia of the mouse is very small and tedious to handle, but the growth data can be easily obtained from a block face which is serially resurfaced and studied by fluorescence CSLM [and BSE SEM as required]. But how might we acquire and handle and orientate real physical sections for more detailed LM analysis? The answer lies with the LAM technique (Figure 14A-C). The sample had been iodine stained for BSE SEM (Figure 14A). LM montages could be matched to previous SEM and CSLM images. The whole tibia TS and its finest internal parts, the medullary trabeculae, were perfectly preserved. It would easily be possible to produce exactly oriented series of LAM sections by controlling the orientation of the block at the moment of gluing it to each slide.

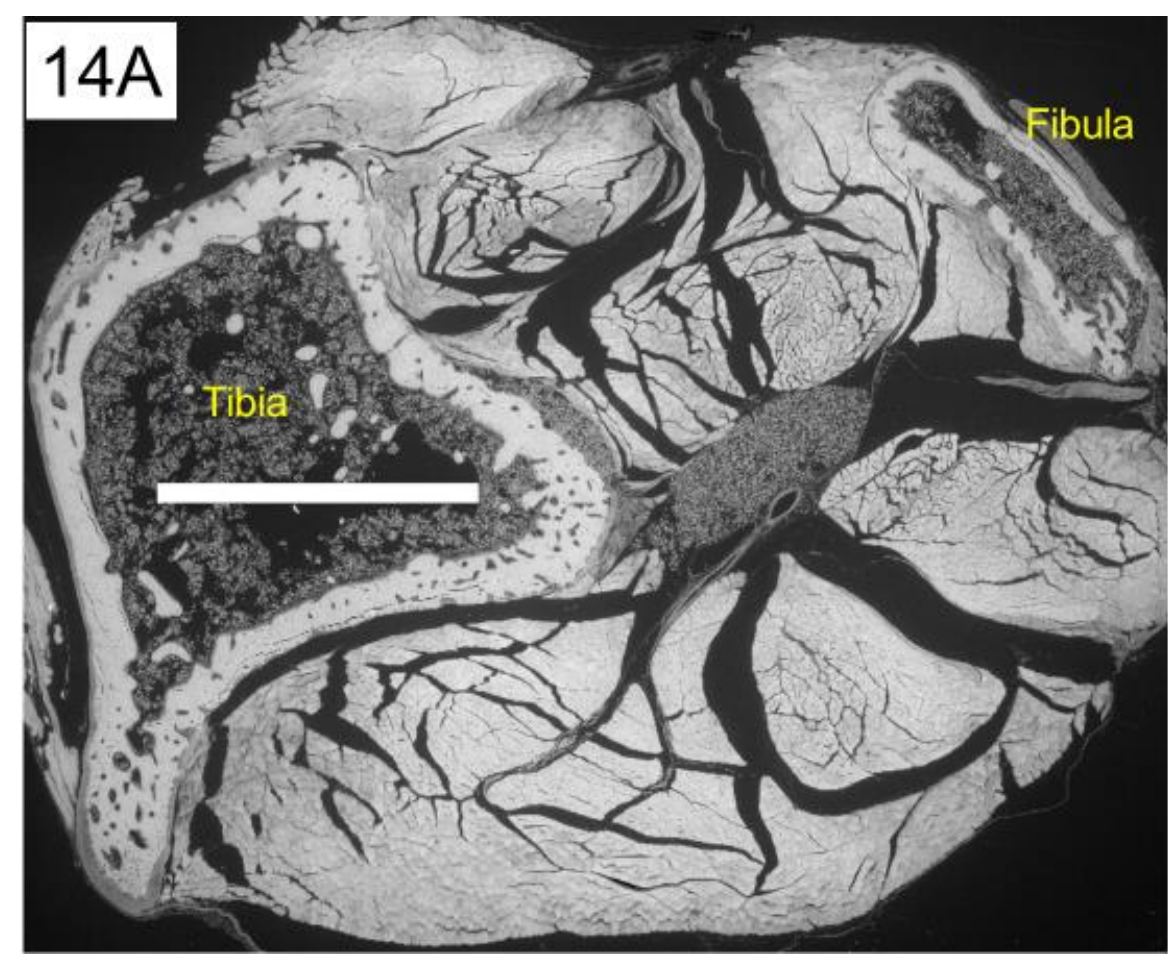




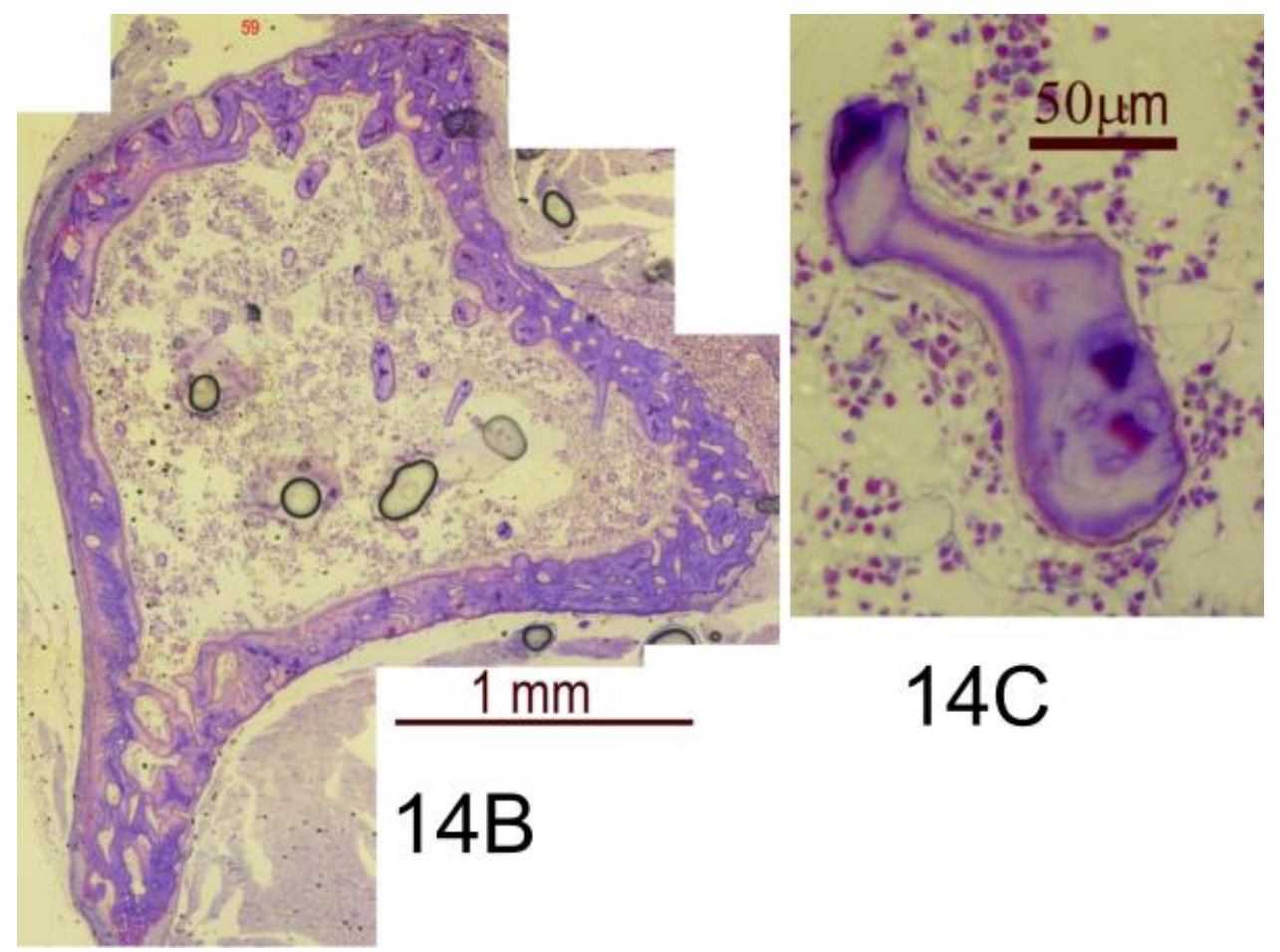

Figure 14A-C. E11 knockout mouse proximal tibia TS. A. Block, iodine vapour stained, BSE SEM, image mirrored to correspond to B, LAM LM stained acridine red and Victoria blue. Dark outlines are air bubbles in glycerol. C. Higher power view of single trabeculum. All parts of section are rigidly held via the presence of the embedding PMMA glued to the glass slide with cyanoacrylate. Scale bar $=1 \mathrm{~mm}$ in A.

\section{Human dental enamel}

The molar LAM LS contained areas of enamel which were very thin section, only one prism thick. Alternate millimetre wide swatches in this section were of different thicknesses, again correlating with changing directions of scanning in Y during the ablation procedure. A polarised light image is shown in Figure 15. 


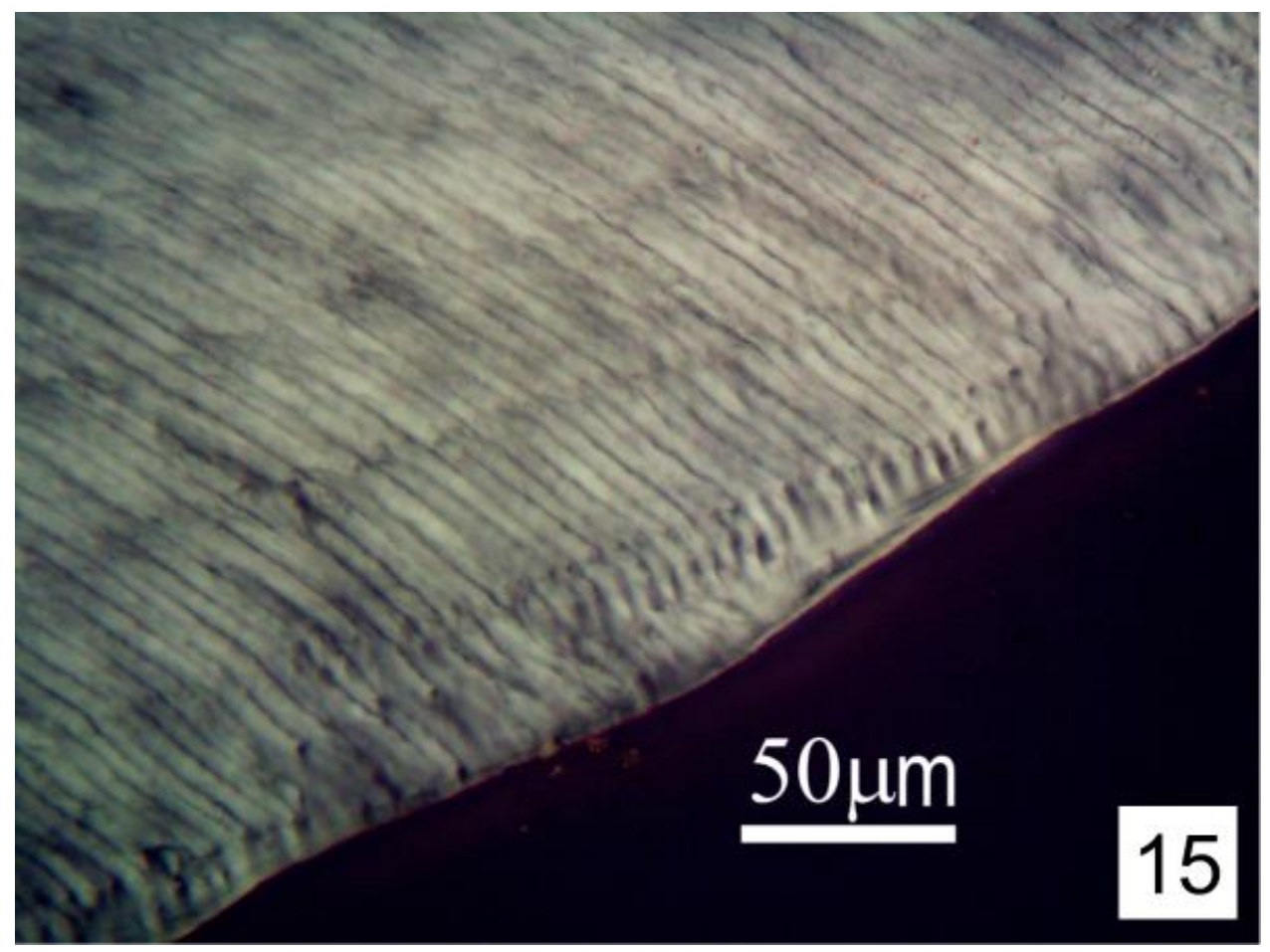

Figure 15. Human third permanent molar LS block face, PLM. Section is only one prism thick.

\section{Discussion}

There are several types of tissues in bones and teeth which provide mechanical support, are normally calcified, hard and tough, and difficult to slice finely - which must be done to understand normal and altered structure and function and relationships to surrounding soft cellular parts which maintain them. Classically, hard tissues are softened by removing the mineral component so that they may be cut with a knife: crystals cannot be so cut, because they cannot be bent. But if we 'decalcify', we remove a main structural component which we need to understand.

Methods for embedding mixed hard and soft tissues in resins to permit thin sectioning with saws followed by serial grinding to produce thin sections are well known in the literature (Gross and Strunz 1977; Donath and Breuner 1982; Plenk 1986; Wolf et al 
1992; Erben 1997; Singhrao et al. 2012). They all aim to make it possible to have thin slices where all the parts are intact and properly connected. The new LAM technology allows us to cut perfect sections by laser machining with the great advantage of minimal wastage. The sections produced are suitable for any form of LM and, because current SEMs make it simple to examine uncoated samples at 50Pa chamber pressure, we can now envisage correlative SEM as a standard method in the LM sphere, and vice versa.

Thin, physical sections retaining the mineral component, but undeformed, are required for full micro-scale characterisation of hard tissues and interfaces to nonmineralised matrices and cells: hydroxy-apatite crystals cannot be bent. The sections produced by laser cutting may be better than those produced by mechanical cutting. They can be thinner than the 'voxel' in most laboratory X-ray microtomography systems. Here, we should bear in mind that the resolution of the light microscope is still far better than that of even the highest resolution, synchotron XMT and this is best obtained from the use of thin sections.

The productivity of laser microtomy is high: one square centimetre blocks can be cut in 10 minutes. Serial slicing can be achieved with as little as 20 microns waste between sections. This is important when dealing with small and valuable samples where we wish to destroy the minimal amount of tissue, and again for small rodent bones which constitute a high proportion of our throughput for genetic manipulation studies in mice. 
For correlative studies with other imaging modalities, including clinical imaging, it is also required that sections can be visualised in the full 3D context of a large volume of the tissue or organ from which they derive. With laser sectioning from PMMA block faces we have a technique in which we get perfect correlation with any microscopic method - including BSE-SEM, qBSE-SEM, iodine stained BSE-SEM, confocal fluorescence or reflection LM - and with X-ray-microtomography. In addition, we create permanent preparations suitable for both LM and SEM. With the new generation of high-specimen-chamber-pressure SEMs, we can regard SEM as a routine extension to the armamentarium of LM methods.

The sections produced by LAM are thin enough to permit application of phase contrast techniques. Here, as in PLM, the limitations are likely to be set by any self structure of the cyano-acrylate adhesive film.

\section{Summary.}

The LAM approach did provide excellent sections from all the tissue types and problems which we chose for this evaluation study. It will make possible the previously impossible. The technique does need some improvement to take care of the problems related to 'snake' scanning, but these are probably trivial.

\section{Acknowledgements}

The samples were provided by - and/or previously co-studied with - several collaborators to whom I am extremely grateful: Christopher M. Riggs, Charles Ley, Sigríður Björnsdóttir, Stina Ekman, Karen Hansson, J.H. Duncan Bassett, Graham R. Williams, James A. Gallagher, Lakshminarayan Ranganath, Roger Smith, Sheila 
Laverty, Nidhi Sofat, Colin Farquharson, Andrew A. Pitsillides, Katherine Staines, Behzad Javaheri, José-Luis Millán, Kevan Wong, Philippe Clezardin and Heiko Richter. I also thank Maureen Arora who embedded all the tissue and prepared the blocks and David Mills and Graham R. Davis who performed the high contrast resolution XMT.

\section{References}

1. Bassett JH et al. 2012a. Mice lacking the calcineurin inhibitor Rcan2 have an isolated defect of osteoblast function. Endocrinology 153:3537-3548.

2. Bassett JH et al. 2012b. Rapid-throughput skeletal phenotyping of 100 knockout mice identifies 9 new genes that determine bone strength. PLoS Genet. 8(8):e1002858. doi: 10.1371/journal.pgen.1002858.

3. Bourassa D, Gleber S-C, Vogt S, Shin CH, Fahrni CJ. 2016. MicroXRF tomographic visualization of zinc and iron in the zebrafish embryo at the onset of the hatching period. Metallomics 8, 1122-1130.

4. Boyde A. 1989. Enamel. In: Handbook of Microscopic Anatomy Vol V/6, pp309-473: A Oksche and L Vollrath (Eds) Springer Verlag, Berlin.

5. Boyde A. 2003. The real response of bone to exercise. J Anat. 203:173-189.

6. Boyde A. 2012. Staining plastic blocks with triiodide to image cells and soft tissues in backscattered electron SEM of skeletal and dental tissues. Eur Cell Mater 24, 154-161.

7. Boyde A et al. 2014a. On fragmenting, densely mineralised acellular protrusions into articular cartilage and their possible role in osteoarthritis. J Anat. 225:436-446. 
8. Boyde A, Mccorkell FA, Taylor GK, Bomphrey RJ, Doube M. 2014b. Iodine vapor staining for atomic number contrast in backscattered electron and X-ray imaging. Microsc Res Tech. 77, 1044-1051.

9. Boyde A, Riggs CM, Bushby AJ, McDermott B, Pinchbeck GL, Clegg PD. 2011. Cartilage damage involving extrusion of mineralisable matrix from the articular calcified cartilage and subchondral bone. Eur Cell Mater. 2011 21:470-478. PMID: 21623571

10. Boyde A, Staines KA, Javaheri B, Millan JL, Pitsillides AA, Farquharson C. 2017. A distinctive patchy osteomalacia characterises Phospho1-deficient mice. J Anat. 231(2):298-308.

11. Boyde, A., Lovicar, L., and Zamecnik, J. (2005) Combining confocal and BSE SEM imaging for bone block surfaces. European Cells \& Materials 26, 33-38.

12. Boyde, A., Travers, R., Glorieux, F. H., and Jones, S. J. (1999) The mineralization density of iliac crest bone from children with osteogenesis imperfecta. Calcif. Tissue Intl. 64, 185-190.

13. Carr AJ, Smith R, Athanasou N, Woods CG. 1995. Fibrogenesis imperfecta ossium. J Bone Joint Surg Br. 77:820-829.

14. Donath K, Breuner G. 1982. A method for the study of undecalcified bones and teeth with attached soft tissues. The Saege-Schliff (sawing and grinding) technique. J Oral Pathol 11:318-326.

15. Erben RG. 1997. Embedding of bone samples in methylmethacrylate: an improved method suitable for bone histomorphometry, histochemistry and immunohistochemistry. J Histochem Cytochem 45: 307-313.

16. Fournier PG, Stresing V, Ebetino FH, Clézardin P. 2010. How do bisphosphonates inhibit bone metastasis in vivo? Neoplasia. 12:571-578. 
17. Gross UM, Strunz V. 1977. Surface staining of sawed sections of undecalcified bone containing alloplastic implants. Stain Technol 52:217-219.

18. Jayasinghe JA, Jones SJ, Boyde A. 1993. Scanning electron microscopy of human lumbar vertebral trabecular bone surfaces. Virchows Arch A Pathol Anat Histopathol. 422:25-34.

19. Kowtharapu BS et al. 2016. J. Microsc. 265, 21-26.

20. Kuttapitiya A et al. 2017. Microarray analysis of bone marrow lesions in osteoarthritis demonstrates upregulation of genes implicated in osteochondral turnover, neurogenesis and inflammation. Ann Rheum Dis. 76:1764-1773.

21. Lacourt M, Gao C, Li A, Girard C, Beauchamp G, Henderson JE, Laverty S. 2012. Relationship between cartilage and subchondral bone lesions in repetitive impact trauma-induced equine osteoarthritis. Osteoarthritis Cartilage. 20:572-583.

22. Laverty S, Lacourt M, Gao C, Henderson JE, Boyde A (2015) High density infill in cracks and protrusions from the articular calcified cartilage in osteoarthritis in standardbred horse carpal bones. Int J Mol Sci. 16:9600-9611.

23. Ley CJ, Ekman S, Hansson K, Björnsdóttir S, Boyde A. 2014. Osteochondral lesions in distal tarsal joints of Icelandic horses reveal strong associations between hyaline and calcified cartilage abnormalities. Eur Cell Mater. 27:213236.

24. Lubatschowski H. 2007. Laser Microtomy. Opt. Photonik 2, 49-51.

25. Pinchbeck GL, Clegg PD, Boyde A, Riggs CM. 2013. Pathological and clinical features associated with palmar/plantar osteochondral disease of the metacarpo / metatarsophalangeal joint in Thoroughbred racehorses. Equine Vet J. 45:587-592. 
26. Plenk H, Jr. 1986. The microscopic evaluation of hard tissue implants. In: Williams DF, editor. Techniques of biocompatibility testing, Vol.2. Boca Raton, FL: CRC Press. pp.35-81.

27. Richter H, Lubatschowski H, Will F. 2011 Laser-Mikrotomie mit ultrakurzen Pulsen - Neue Perspektiven für die Gewebe- und Biomaterialbearbeitung. BioPhotonik 2, 50-52.

28. Richter H, Ojeda DFR, Will F. 2014 Lasergesteuerte Probenpräparation von Hartgeweben und Biomaterialen. BIOspektrum 20, 538-539.

29. Schimek K et al. 2013 Integrating biological vasculature into a multi-organchip microsystem. Lab. Chip 13, 3588.

30. Singhrao SK, Nicholson K, Crean S. 2012. Informed choices for challenging specimens when choosing methacrylate resin systems for histology. Microsc Res Technique 75:576-585.

31. Staines KA et al. 2017. Hypomorphic conditional deletion of E11/Podoplanin reveals a role in osteocyte dendrite elongation. J Cell Physiol. 232:3006-3019.

32. Staines KA, Prideaux M, Allen S, Buttle DJ, Pitsillides AA, Farquharson C. 2016. E11/Podoplanin protein stabilization through inhibition of the proteasome promotes osteocyte differentiation in murine in vitro models. $\mathbf{J}$ Cell Physiol. 231:1392-1404.

33. Taylor AM, Boyde A, Davidson JS, Jarvis JC, Ranganath LR, Gallagher JA. 2012. Identification of trabecular excrescences, novel microanatomical structures, present in bone in osteoarthropathies. Eur Cell Mater. 23:300-309.

34. Will F, Richter H. 2015 Laser-based Preparation of Biological Tissue. Laser Tech. J. 12, 44-47. 
35. Wolf E, Roeser K, Hahn M, Welkerling H, Delling G. 1992. Enzyme and immunohistochemistry on undecalcified bone and bone marrow biopsies after embedding in plastic: A new embedding method for routine application. Virchows Arch [A] 420:17-24.

36. Wong K. 2000. Studies of the quality of the intraosseous dental implant bed and of thermal effects in implant pathology. PhD Thesis. Dept. of Anatomy and Developmental Biology, University College London.

37. Zhang K et al. 2006. E11/gp38 selective expression in osteocytes: regulation by mechanical strain and role in dendrite elongation. Molecular and Cellular Biology. 26:4539-4552. 


\section{List of abbreviations used in text and figures}

ACC Articular calcified cartilage

AKU Alkaptonuria

BSE BackScattered Electron

FC fibrocartilage

FIO Fibrogenesis imperfecta ossium

HAC Hyaline articular cartilage

HDMI High density mineralised infill

HDMP High density mineralised protrusion from ACC MF into HAC

LAM Laser ablation microtomy /machined / machining

LM Light microscope / microscopy

MAR Mineral Apposition Rate (by double labelling, surrogate for growth rate at a surface)

MF Mineralising front or Mineral front (present tidemark border ACC-HAC)

OA Osteoarthritis

OB osteoblast

PLM Polarised light microscope / microscopy [crossed linear polarisers]

PMMA poly-methyl-methacrylate

POD Palmar osteochondral disease

qBSE quantitative backscattered electron imaging using standards

SCB Subchondral bone

SEM scanning electron microscope / microscopy

TB Thoroughbred horse

XMT ( $\mu \mathrm{CT}$, micro CT) X-ray microtomography 


\section{Second Abstract -- Lay Description}

There are several types of tissues in bones and teeth which provide mechanical support, are normally calcified, hard and tough, and difficult to slice finely - which must be done to understand normal and altered structure and function and relationships to surrounding soft cellular parts which maintain them. Classically, hard tissues are softened by removing the mineral component so that they may be cut with a knife: crystals cannot be so cut, because they cannot be bent. But if we 'decalcify', we remove a main structural component which we need to understand. It has been next to impossible to have thin slices where all the parts are intact and properly connected.

We investigated cutting sections by laser machining. In extensive pilot studies, we have shown that the method works to produce slide-mounted samples which we can study by both light and electron microscopy. The sections produced are thinner than the 'voxel' in most laboratory X-ray microtomography systems. It should be born in mind that the resolution of the light microscope is still far greater than that of even the highest resolution, synchotron XMT and that this is best obtained in the present context from the use of thin sections. Serial slicing can be achieved with as little as 20 microns waste between sections. Productivity is high: one square centimetre blocks can be cut in 10 minutes.

The basic procedure tested to date has used PMMA embedded material, but this could be any resin, including LR White and glycol methacrylate. A flat block face is produced by micromilling or polishing. The block face can be studied by SEM and confocal at this stage. The block face and the underlying few microns are to be contained in the first section. The block face is stuck to a standard glass light microscope slide with a cyanoacrylate adhesive. We focus through the glass slide, the 
cyanoacrylate adhesive, the block face, and a further increment into the specimen. The focussed, pulsed laser beam is scanned along a $1 \mathrm{~mm}$ line, and this swathe is translated in an XY snake scan to cover the entire area of the block, which is thereby released from the slide, leaving the section stuck to the slide. Light, wet polishing on the finest grade (4000 grit) silicon carbide polishing paper is used to remove a 1-2 $\mu \mathrm{m}$ thick damaged layer at the surface of the section. 